\title{
Effect of Channeling Agents on the Release Profile of Theophylline from METHOCEL K4M Based Matrix tablets
}

\author{
Md. Shaikhul Millat Ibn Razzak, Ferdous Khan, Md. Ziaur Rahman Khan, \\ Kanij Fatema, Muhammed Shahidul Islam and Md. Selim Reza
}

\author{
Department of Pharmaceutical Technology, Faculty of Pharmacy, University of Dhaka, \\ Dhaka-1000, Bangladesh
}

\begin{abstract}
The present study was undertaken to investigate the effect of channeling agents on the release profile of Theophylline from METHOCEL K4M based matrix systems. Matrix tablets of Theophylline using METHOCEL $\mathrm{K} 4 \mathrm{M}$ were prepared by direct compression process. METHOCEL K4M polymer is hydrophilic in nature. $\mathrm{NaCl}$ and PEG 1500 were used as channeling agents. Drug release study was evaluated for eight hours using USP 22 paddletype dissolution apparatus using distilled water as the dissolution medium. The release mechanisms were explored and explained with zero order, Higuchi and Korsmeyer equations. The release rate, extent and mechanisms were found to be governed by channeling agent type and content. Higher channeling agent content $(42.49 \%)$ in the matrix increased the rate and extent of the drug release because of increased porosity in the tablet matrices, at lower channeling agent $(19.76 \%)$ level, the rate and extent of drug release was decreased and in absence of channeling agents these were least. $\mathrm{NaCl}$ ensures maximum release of drug from low viscosity grade METHOCEL K4M than PEG 1500 when other parameters were kept constant. It was found that type and amount of channeling agent significantly affect the time required for $50 \%$ of drug release $\left(\mathrm{T}_{50 \%}\right)$, percentage drug release at 8 hours, release rate constant $(\mathrm{K})$ and diffusion exponent $(\mathrm{n})$. Kinetic modeling of dissolution profiles revealed drug release mechanism ranges from diffusion controlled or Fickian transport to anomalous type or non-Fickian transport, which was mainly dependent on the presence of type and amount of channeling agent. These studies indicate that the proper balance between a matrix forming agent and a channeling agent can produce a drug dissolution profile similar to a theoretical dissolution profile.
\end{abstract}

Key words: Channeling agent, Theophylline, Release Profile, Methocel K4M

\section{INTRODUCTION}

The drug delivery technology landscape is highly competitive and rapidly evolving. Drug dosage forms are keeping pace with high-tech times. In recent years, there have been numerous developments in polymeric carriers and controlled release systems such as films with drug in a Polymer Matrix / Monolithic Devices, ${ }^{1}$ the drug contained by the polymer that acts as Reservoir Devices, ${ }^{2}$ polymeric

Correspondence to: Md. Selim Reza

Tel: 880-2-8612069; Fax: 880-2-8615583

E-mail: selimreza_04@yahoo.com

Dhaka Univ. J. Pharm. Sci. 7(1): 27-32, 2008 (June) colloidal particles or microencapsulates (Microspheres or Nanoparticles) in the form of reservoir and matrix devices, ${ }^{3}$ drug contained by a polymer containing a Hydrophilic or Leachable Additive eg, a second polymer, surfactant or plasticizer, etc., ${ }^{4-6}$, enteric coatings ionize \& dissolve at suitable $\mathrm{pH}^{7}$ soluble polymers with covalently attached 'Pendant' Drug molecules, ${ }^{8,9}$ devices where release rate is controlled dynamically, eg, the Osmotic Pump. ${ }^{10}$

The matrix tablet describes a tablet in which the drug is applied in a skeleton of nondissolving material. It needs simply direct compression of 
blended drugs and retarding additives to form tablets. It is one of the least complicated approaches to the manufacture of sustained/controlled release dosage forms, which consists of a drug dispersed in a polymer, the polymer playing the role of a matrix. ${ }^{11-14}$

\section{MATERIALS AND METHODS}

Theophylline was a gift sample from Square Pharmaceuticals Bangladesh Limited. Colorcon, USA was the source of METHOCEL K4M while PEG 1500 was received from BASF Bangladesh Ltd. $\mathrm{NaCl}$ was obtained from Loba Cheme Pvt. Ltd., India. Aerosil 200 and Magnesium stearate were procured from Degussa, Germany and Wilfrid Smith Ltd. UK. respectively.

Preparation of matrix tablets of Theophylline. Before tablet preparation both the channeling agents were analysed by different mesh size sieves. After thorough experimentation substantial amount of particles were retained in a 40/50 sieve combination. The particles were passed through 40 mesh and retained on 50 mesh sieve which gave the particle size in a range of $297 \mu \mathrm{m}-420 \mu \mathrm{m} .{ }^{15}$ During tablet preparation, method of dry blending of the active ingredients with polymer, filler, lubricant and flow promoter followed by direct compression was adopted. The active ingredient and other excipients were accurately weighted for thirty tablets according to the formulations. Properly weighed METHOCEL $\mathrm{K} 4 \mathrm{M}$, sodium chloride or PEG 1500, magnesium stearate, aerosil 200 and the active ingredient were then taken in a photo film container and blended in a laboratory designed small drum blender for 30 minutes. Particular attention has been given to ensure thorough mixing and phase homogenization. The appropriate amounts of the mixture were accurately weighted in an electronic balance for the preparation of each tablet and finally compressed using a PerkinElmer laboratory hydraulic press equipped with an $11.7 \mathrm{~mm}$ flat faced punch and die set. The compression force and compression time were 5 ton and 30 seconds respectively. Before compression, the surfaces of the die and punch were lubricated with magnesium stearate. All the preparations were stored in airtight containers at room temperature for further study.

In vitro Release Studies of Theophylline. The release rate of theophylline from matrix tablets was determined by using Tablet Dissolution Tester USP XXII. The dissolution test was performed using 900 $\mathrm{ml}$ distilled water at $37 \pm 0.5^{\circ} \mathrm{C}$ and 50 r.p.m. At every 1 hour interval samples of $10 \mathrm{ml}$ were withdrawn from the dissolution medium and that amount was replaced with fresh medium to maintain the volume constant. The samples were filtered through a Whatmaan filter paper and diluted to a suitable concentration with distilled water. The absorbance of the solutions was measured at $271 \mathrm{~nm}$ for drug theophylline by using a Shimadzu UV-1201 $\mathrm{UV} / \mathrm{Vis}$ double beam spectrophotometer (Shimadzu, Japan). Percentage of drug release was calculated using an equation obtained from the standard curve. The dissolution study was continued for 8 hours to get a simulated picture of the drug release in the in vivo condition and drug dissolved at specified time periods was plotted as percent release versus time (hours) curve. This drug release profiles were fitted with several mathematical models to get an idea about the release mechanism of Theophylline from the matrix tablets.

Kinetic modeling of drug release. After completing in vitro dissolution of all the batches for eight hours, the data was treated with zero order, ${ }^{16}$ Higuchi ${ }^{17}$ equations (equation 1-2 respectively).

$$
\begin{aligned}
M_{t}= & M_{0}+k_{0} t \ldots \ldots \ldots \ldots \ldots \ldots \ldots \ldots(1) \\
& M_{t}=M_{0}-k_{H} t^{1 / 2} \ldots \ldots \ldots \ldots \ldots \ldots \ldots . .(2)
\end{aligned}
$$

In these equations, $M_{t}$ is the cumulative amount of drug released at any specified time ( $\mathrm{t}$ ) and $\mathrm{M}_{0}$ is the dose of the drug incorporated in the delivery system. $\mathrm{k}_{0}$ and $\mathrm{k}_{\mathrm{H}}$ are rate constants for zero order and Higuchi model respectively. These models fail to explain drug release mechanism due to swelling (upon hydration) along with gradual erosion of the matrix. Therefore the dissolution data were also fitted to well-known Korsmeyer $^{18}$ kinetic equation to ascertain the mechanism of drug release.

$$
\log \left(M_{t} / M_{\infty}\right)=\log k+n \log t \ldots \ldots \ldots \ldots \ldots(3)
$$


Where, $\mathrm{M}_{\infty}$ is the amount of drug release after infinite time; $\mathrm{k}$ is the release rate constant which considers structural and geometric characteristics of the tablet; and $\mathrm{n}$ is the diffusional exponent or release exponent; indicative of the mechanism of drug release. For a tablet having cylindrical shape, when $\mathrm{n}$ is bellow 0.45, the Fickian diffusion phenomenon dominates, and $\mathrm{n}$ between 0.45 and 0.89 is an anomalous transport (non-Fickian diffusion), often termed as first-order release. After the $\mathrm{n}$ value reaches 0.89 and above, the release can be characterized by case II and super case II transport, which means the drug release rate does not change over time and the release is characterized by zeroorder release. In this case, the drug release is dominated by the erosion and swelling of the polymer. ${ }^{19-20}$ Mean dissolution time (MDT) was calculated from dissolution data using the following equation: ${ }^{16}$

$$
M D T=\left(\frac{n}{n+1}\right) k^{-1 / n}
$$

\section{RESULTS AND DISCUSSION}

To investigate the effect of channeling agents $(\mathrm{NaCl}, \mathrm{PEG} 1500)$ on Theophylline release seven formulations were made (Table 1). Formulation-1 best fits with Korsmeyer $\left(\mathrm{R}^{2}=0.993\right)$ and zero order $\left(\mathrm{R}^{2}=0.993\right)$ kinetic models to the same extent. The value of release exponent 0.771 which indicates that the release pattern of theophylline from Formulation1 was followed anomalous transport mechanism, which appears to indicate a coupling of the diffusion and erosion mechanism. ${ }^{18}$ Formulation-2, Formulation-3 and Formulation-4 also followed Korsmeyer model $\left(\mathrm{R}^{2}=0.993, \mathrm{R}^{2}=0.996\right.$ and $\mathrm{R}^{2}=$ 0.994). The values of $\mathrm{n}$ for Formulation-2 and Formulation-3 are 0.644 and 0.614 respectively (Table 2) which indicate that the drug was released by anomalous transport. But the value of release exponent for Formulation-4 is 0.360. This value indicates that the drug was released from Formulation-4 by following Fickian release pattern, more specifically diffusion controlled release mechanism which means the zero order release rate was changed over time. ${ }^{18}$ The release can be poorly explained by zero order release profile which is supported by the $\mathrm{R}$ squared value (0.825) of Formulation-4. In the same manner Formulation-5 and Formulation- 6 followed Korsmeyer model $\left(\mathrm{R}^{2}=\right.$ 0.996 and $\mathrm{R}^{2}=0.998$ ) predominantly and whereas Formulation-7 primarily followed Higuchian release pattern $\left(\mathrm{R}^{2}=0.998\right)$ and secondarily follows Korsmeyer model $\left(R^{2}=0.997\right)$. The values of $n$ for Formulation-5, Formulation-6 and Formulation-7 are $0.679,0.629$ and 0.473 respectively that indicate the drug was released by anomalous transport (Table 2). For each channeling agent the values of diffusion exponent (n) was reduced with the increase of channeling agent load which indicates the shifting of release mechanism from non- Fickian transport to Fickian transport in case of $\mathrm{NaCl}$ and shifting towards Fickian transport in case of PEG 1500. This effect is due to the formation of channels that facilitate the diffusion mechanism.

From the Table 2, it is clear that $\mathrm{T}_{50 \%}$ (MDT) values were changed due to the change of the amount of channeling agents in the matrix tablets. In all these formulations the values of $\mathrm{T}_{50 \%}$ (MDT) are larger for those formulations which contain smaller quantities or absence of channeling agents. For example $\mathrm{T}_{50 \%}$ (MDT) values for Formulation-1, Formulation-2, Formulation-3 and Formulation-4 are 11.2 hours, 7.4 hours, 5.5 hours and 2.3 hours respectively. This reduction of the magnitude of $\mathrm{T}_{50 \%}$ (MDT) is most valuable observation which indicates that when the amount of $\mathrm{NaCl}$ was increased the drug release rate was increased gradually due to the channeling effect of the $\mathrm{NaCl}$ and same picture was observed in case of Formulation-5, Formulation-6 and Formulation-7 where PEG 1500 used as channeling agent.

Effect of channeling agent. Formulation-4 and Formulation-7 were compared to investigate the effect of the channeling agent ( $\mathrm{NaCl}$ and PEG 1500) in METHOCEL K4M matrices. Formulation-4 and Formulation-7 contained $300 \mathrm{mg} \mathrm{NaCl}$ and PEG 1500 respectively keeping other parameters constant. From the zero order release profile it is observed that 
the total percent release of theophylline from Formulation-4 and Formulation-7 were $79.5 \%$ and $74.2 \%$ respectively at the end of eight hour (Table 2 ). From the Figure 1, it is found that the release rate of theophylline governed by $\mathrm{NaCl}$ and PEG 1500 was more or less same but the extent of release of drug was rapid and more in case of $\mathrm{NaCl}$ than PEG 1500. Most of the formulations followed anomalous transport mechanism as well as some of them followed diffusion dominated mechanism at high amount of $\mathrm{NaCl}$ and PEG 1500.

Table 1. Composition of different formulations of matrix tablets (in mg)

\begin{tabular}{lcccccc}
\hline Formulation & Theophylline & METHOCEL K4M & $\mathrm{NaCl}$ & PEG 1500 & Aerosil 200 & Mg-stearate \\
\hline Formulation-1 & 300 & 100 & - & - & 4 & 2 \\
Formulation-2 & 300 & 100 & 100 & - & 4 & 2 \\
Formulation-3 & 300 & 100 & 200 & - & 4 & 2 \\
Formulation-4 & 300 & 100 & 300 & - & 4 & 2 \\
Formulation-5 & 300 & 100 & - & 100 & 4 & 2 \\
Formulation-6 & 300 & 100 & - & 200 & 4 & 2 \\
Formulation-7 & 300 & 100 & - & 300 & 4 & 2 \\
\hline
\end{tabular}

Table 2. Release rate constants and R-squared values for different release kinetics as well as Successive fractional dissolution time of seven formulations of Theophylline matrix tablets

\begin{tabular}{|c|c|c|c|c|c|c|c|}
\hline \multirow{2}{*}{ Formulation } & \multicolumn{2}{|c|}{ Zero Order } & \multicolumn{2}{|c|}{ Highuchi } & \multicolumn{2}{|c|}{ Korsmeyer } & \multirow{2}{*}{$\begin{array}{c}\text { Fractional dissolution } \\
\text { time }\end{array}$} \\
\hline & $\mathrm{K}_{\mathrm{o}}$ & $\mathrm{R}^{2}$ & $\mathrm{~K}_{\mathrm{h}}$ & $\mathrm{R}^{2}$ & $\mathrm{n}$ & $\mathrm{R}^{2}$ & \\
\hline Formulation-1 & 4.83 & 0.993 & 14.37 & 0.945 & 0.771 & 0.993 & 11.2 \\
\hline Formulation-2 & 6.33 & 0.976 & 19.27 & 0.975 & 0.644 & 0.993 & 7.4 \\
\hline Formulation-3 & 7.45 & 0.968 & 22.90 & 0.985 & 0.614 & 0.996 & 5.5 \\
\hline Formulation-4 & 8.11 & 0.825 & 26.92 & 0.979 & 0.360 & 0.994 & 2.3 \\
\hline Formulation-5 & 6.07 & 0.982 & 18.41 & 0.972 & 0.679 & 0.996 & 8.0 \\
\hline Formulation-6 & 7.08 & 0.964 & 21.84 & 0.988 & 0.629 & 0.998 & 5.9 \\
\hline Formulation-7 & 8.08 & 0.895 & 26.01 & 0.998 & 0.473 & 0.997 & 3.5 \\
\hline
\end{tabular}

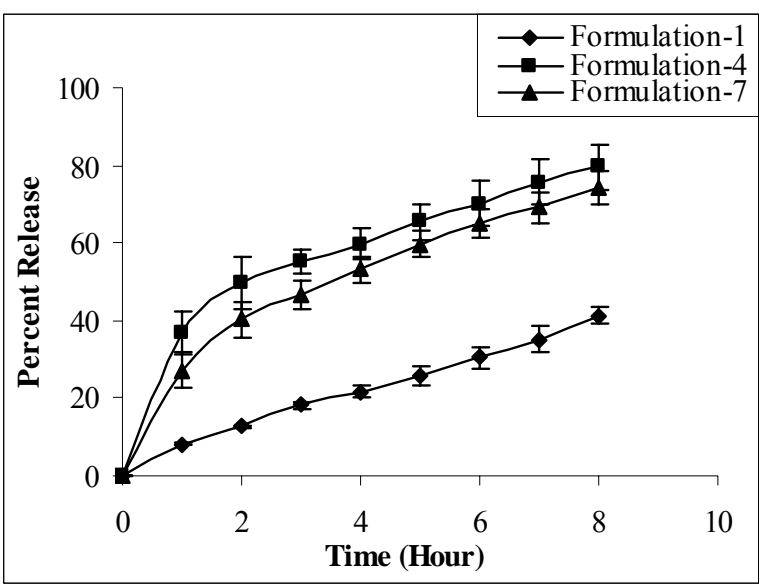

Figure 1. Variation of drug release due to the change of channeling agents type (NaCl, PEG 1500) (c) $300 \mathrm{mg}$

Effect of amount of channeling agents ( $\mathrm{NaCl}$, PEG 1500). From the zero order release profile it is observed that the total percent release of theophylline from Formulation-1, Formulation-2, Formulation-3,
Formulation-4 were $41.3 \%, 55.2 \%, 64.1 \%, 79.5 \%$ and from Formulation-5, Formulation-6, Formulation- 7 were $51.8 \%, 61.1 \%$ and $74.2 \%$ respectively at the end of eight hour (Table 2). From the Figure 2, it is observed that without channeling agent, drug release from the Formulation-1 was slow. This effect was due to the characteristic property of METHOCEL K4M to form gels in situ. This type of polymers forms a gel like layer around the matrix system. The rate and extent of theophylline release increases from the matrices with increasing the amount $\mathrm{NaCl}$ in the Formulation-2, Formulation-3 and Formulation-4 (Figure 2a) and the amount of PEG 1500 in the Formulation-5, Formulation-6 and Formulation-7 (Figure 2b). The addition of channeling agents deviate the formulations to follow zero order kinetic model (Table 2). 

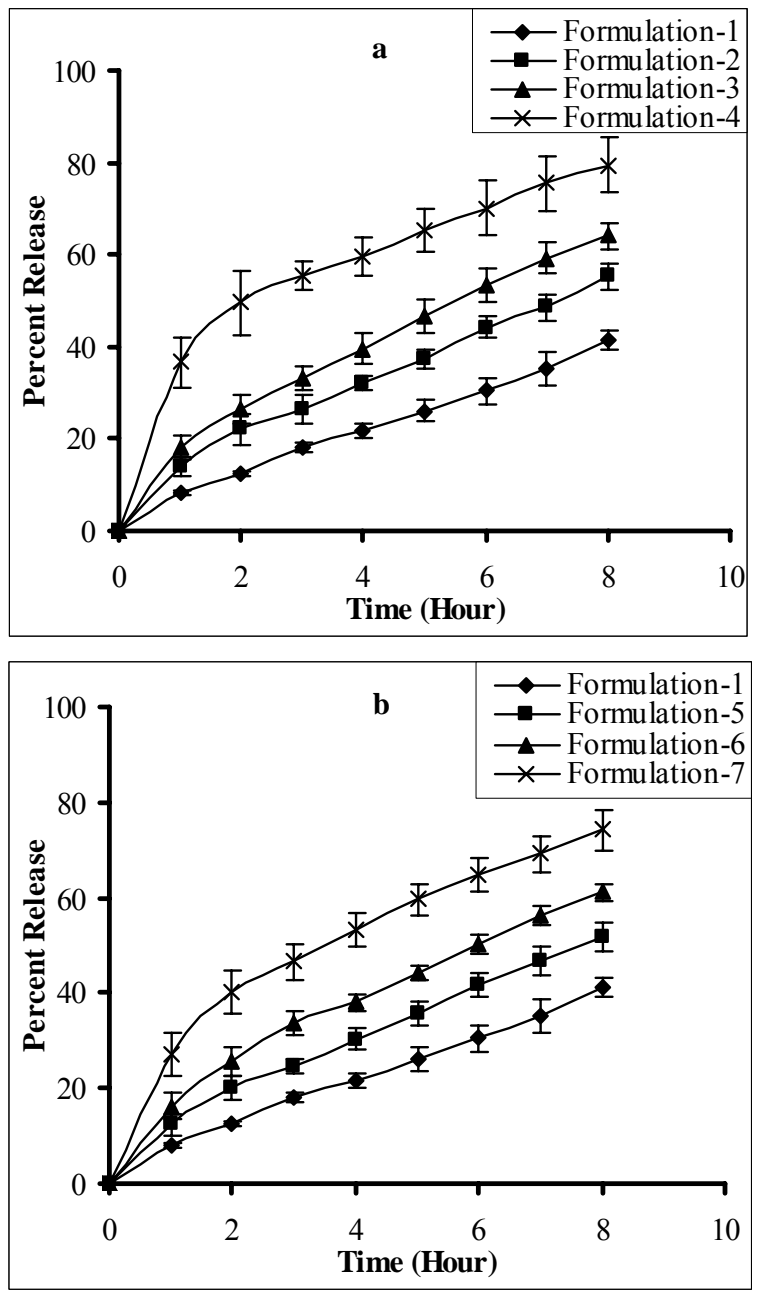

Figure 2. Variation of drug release due to the change of amount of channeling agents from METHOCEL K4M (a) $\mathrm{NaCl}$, (b) PEG 1500

In all these cases the increase of the amount of $\mathrm{NaCl}$ and PEG 1500 cause a gradually more release of drug in dissolution study which is supported by the values of $\mathrm{T}_{50 \%}$. From the Figure 2 another observation was that, when the amount of channeling agent was increased, the control of the polymer upon the release profile was to some extent lost which is demonstrated by the standard error bars parallel to Yaxis. Conversely it can be said that at relatively higher polymer contents (considering polymer to $\mathrm{NaCl}$ ratio) the drug release pattern becomes slow but steady. From the above formulations it can be concluded that a suitable combination of METHOCEL K4M and channeling agents $(\mathrm{NaCl}$ and
PEG 1500) can give us matrix system with desirable drug release profile.

\section{CONCLUSION}

Channeling agents variation both amount and type significantly affect drug release kinetics from prepared matrix tablets. Higher amount of channeling agent increases drug release rate and extent irrespective of its type. Most of the formulations fit better with Korsmeyer equation which defined drug release rate and mechanism. With the increase of channeling agent content, maximum formulations deviates to follow zero order release kinetics. In all cases the increase of the channeling agent content causes a lowering of the magnitude of release exponent (n) which indicates the shifting of release mechanism from non-Fickian to Fickian direction. At lower amount, $\mathrm{NaCl}$ and PEG 1500 exert almost same channeling effect but while increasing amount of channeling agent, $\mathrm{NaCl}$ showed greater release than PEG 1500. If the facility of scanning electron micrograph (SEM) is available then it will be possible for us to take the picture of the pores formed by channeling agents. In future we have a plan to observe such effects with different release rate retarding polymers.

\section{REFERENCES}

1. Singh, P., Desai, S.J., Simonelli, A.P. and Higuchi, W.I. 1988. Role of Wetting on the Rate of Drug Release from Inert Matrices. J. Pharm. Sci. 57, 217-226.

2. Nakagami, H., Keshikawa, T., Matsumura, M. and Tsukamoto H. 1991. Application of aqueous suspensions and latex dispersions of water-insoluble polymers for tablet and granule coating. Chem. Pharm. Bull. 39, 1837-1842.

3. Giddings, A.E.B. Farquharson-Roberts, M.A. and Nunn, A.J. 1975. Perforation of small bowel due to slow release potassium chloride (slow-K). Brit. Med. J. 206.

4. Lordi, N.G. 1987. The theory and practice of industrial pharmacy, $3^{\text {rd }}$ edition, LEA \& FEBIGER Philadelphia, USA, pp. $430-456$.

5. Udeala, O.K. and Aly, S.A.S. 1989. Degradation kinetics of thiamine hydrochloride in directly compressed tablets III. water vapour transmission through free and applied Eudragit films. Drug Dev. Ind. Pharm. 15, 1797-1825. 
6. Gardner, C. 1983. Drug targeting: potentials and limitations, In: Briemer, D. D. and Speiser, P. (Eds.), Pharmaceutical Science, pp. 291-303.

7. Gregoriadis, G., Senior, J. and Poste, G. (Eds.). 1986. Targeting of drugs with synthetic systems. NATO-ASI Series (No. 113). Plenum Press, New York.

8. Poznansky, M.J. and Juliano, R.L. 1984. Biological approaches to the controlled delivery of drugs: a Critical Review. 36, 236 - 277.

9. Tomlinson, E. and Davis, S.S. (Eds). 1986. Site specific drug delivery, Wiley Int., Chichester.

10. O'Donnell, P.B. and McGinity, J.W. 1997. Preparation of microspheres by the solvent evaporation technique. Advanced Drug Delivery Reviews 28, 25-42.

11. Armand, J.Y., Magnard, F., Bouzon, J., Rollet, M., Taverdet, J.L. and Vernaud, J.M. 1987. Modeling of the release of drug in gastric fluid from spheric galenics form with Eudragit matrix. Int. J. Pharm. 40, 33-41.

12. Fessi, H., Marty, J.P., Puiseiux, F. and Carstensen, J.Y. 1982. Square root of time dependence of matrix formulations with low drug content. J. Pharm. Sci. 714, 749-752.

13. Focher, B., Marzetti, A., Sarto, V., Balltrame, P.L. and Carmitti, F. 1984. Cellulosic materials: structure and enzymatic hydrolysis relationships. J. Appl. Polym. Sci. 29, 3329-3338.
14. Heller, J. 1984. Biodegradable polymers in controlled drug delivery. CRC Crit. Rev. Therm. Drug Carrier Syst. 1, 39-90.

15. Lachman, L., Lieberman, H.A. and Kanig, J. L. (eds.). 1990. Sustained Release Dosage Forms. In: The Theory and Practice of Industrial Pharmacy, $3^{\text {rd }}$ edition, Varghese Publishing House, Bombay, pp. 430-456.

16. Mockel, J.E. and Lippold, B.C. 1993. Zero order release from hydrocolloid matrices. Pharm. Res. 10, 1066-1070.

17. Higuchi, T. 1963. Mechanism of sustained action medication, theoretical analysis of rate of release of solid drugs dispersed in solid matrices. J. Pharm. Sci. 52, 1145-1149.

18. Korsmeyer, R.W., Gurny, R., Doelker, E., Buri, P. and Peppas, N.A. 1983. Mechanisms of solute release from porous hydrophilic polymers. Int. J. Pharm. 15, 25-35.

19. Peppas, N.A. 1985. Analysis of Fickian and non-Fickian drug release from polymers. Pharm. Acta. Helv. 60, 110-111.

20. Chueh, H.R., Zia, H. and Rhodes, C.T. 1995. Optimization of sotalol floating and bioadhesive extended release tablet formulations. Drug Dev. Ind. Pharm. 21, 1725-1747. 